\title{
Second-Order Time Correlations within a Polariton Bose-Einstein Condensate in a CdTe Microcavity
}

\author{
J. Kasprzak, ${ }^{1, *}$ M. Richard, ${ }^{2}$ A. Baas, ${ }^{2}$ B. Deveaud,${ }^{2}$ R. André, ${ }^{1}$ J.-Ph. Poizat, ${ }^{1}$ and Le Si Dang ${ }^{1}$ \\ ${ }^{1}$ Institut Néel, CNRS/UJF, 25 avenue des Martyrs, BP 16638042 Grenoble cedex 9, France \\ ${ }^{2}$ Ecole Polytechnique Fédérale de Lausanne (EPFL), Station 3, CH-1015 Lausanne, Switzerland
}

(Received 2 May 2007; published 14 February 2008)

\begin{abstract}
Second-order time correlations of polaritons have been measured across the condensation threshold in a CdTe microcavity. The onset of Bose-Einstein condensation is marked by the disappearance of photon bunching, demonstrating the transition from a thermal-like state to a coherent state. Coherence is, however, degraded with increasing polariton density, most probably as a result of self-interaction within the condensate and scatterings with noncondensed excitons and polaritons. Such behavior clearly differentiates polariton Bose condensation from photon lasing.
\end{abstract}

PACS numbers: 78.67.De, 03.75.Nt, 78.67.Hc, 78.70.--g

Bose-Einstein condensation (BEC) is a thermodynamic phase transition occurring upon increasing the density of bosons, leading to the massive occupation of the lowest quantum state and the onset of a long range order parameter $[1,2]$. These two BEC defining features were clearly observed in dilute atomic gases when lowering their temperature down to about $10^{-6} \mathrm{~K}$ [3-6]. Further supports of condensation were provided by second and third order correlation studies of atom condensates [7-10]. For solid state systems, $\mathrm{BEC}$ has been claimed for excitons in $\mathrm{Cu}_{2} \mathrm{O}$ [11-13], coupled semiconductor quantum wells (QWs) [14,15], and quantum Hall bilayers [16], as well as for magnons [17], and for exciton polaritons in semiconductor microcavities $[18,19]$. However, it is only in the polariton system that both BEC signatures, namely, the massive occupation of the ground state and the onset of long range spatial coherence, have been demonstrated [19]. Exciton polaritons are two-dimensional quasi particles, which result from the strong coupling between excitons confined in the QWs and photon modes confined in the microcavity $[20,21]$. Of particular interest for BEC is the extremely light polariton effective mass, around $10^{-4}$ the free electron mass, which should allow condensation at the bottom of the lower dispersion branch up to $300 \mathrm{~K}$ in wide-bandgap semiconductor and organic microcavities [21].

Polariton condensation in CdTe is far from the ideal BEC case since polaritons have a short lifetime (1-2 ps) and show strong interactions between them and their environment through the excitonic part of the polariton. Thus, decoherence could be expected in the condensed phase due to Coulomb scattering of polaritons within the condensate and with noncondensed excitons and polaritons. Indeed phase diffusion is predicted to dominate in the condensed phase, so that the linewidth of the condensate emission would increase rapidly with increasing condensed polaritons [22]. Such degradation of coherence, which would be absent in a standard photon laser, has been recently reported in a CdTe microcavity [19]. On the other hand, polaritons in GaAs microcavity display a quite different behavior [18]. Second-order time correlation measurements showed that fluctuations in the polariton system steadily decrease when increasing the density up to 17 times the condensation threshold density. The purpose of this Letter is to probe second-order time correlations in the same CdTe microcavity as recently used to demonstrate BEC [19]. This measurement provides a direct insight into the quantum fluctuations of the condensate, which we compare with the measurements reported in [18], and with the well-known fluctuations of a photon laser.

In this work, we have measured the second-order time correlations of the microcavity emission, under both $\mathrm{cw}$ and pulsed excitations, using a Hanbury Brown and Twiss (HBT) setup [23]. In cw experiments, we observe photon bunching for excitation below the condensation threshold, indicating the thermal character of the polariton system. Photon bunching is suppressed for excitation just above threshold, demonstrating the formation of a coherent state and confirming the recent BEC claim [19]. With further increase of the excitation intensity, photon bunching occurs again. In pulsed experiments, for excitation far above threshold, we measure a strong increase of fluctuations, which confirms the phase induced decoherence already observed in cw experiments.

The experimental conditions of this work are similar to those described in [19]. We use the same $2 \lambda$ microcavity containing $16 \mathrm{CdTe} / \mathrm{CdMgTe} 50 \AA \mathrm{QWs}$, which exhibits a Rabi splitting of $26 \mathrm{meV}$ [24]. Experiments are carried out at a sample temperature of $5 \mathrm{~K}$ and for photon-exciton detuning of $+2 \mathrm{meV}$. We excite the microcavity with a linearly polarized light at $1.77 \mathrm{eV}$ provided by a Ti:sapphire laser, approximately $100 \mathrm{meV}$ above the lower polariton ground state. The excitation spot has a homogenous "top hat" intensity profile of around $30 \mu \mathrm{m}$ diameter. The exciton density at the condensation threshold per QW is about 50 times below the exciton saturation density in CdTe $\left(\sim 5 \times 10^{11} \mathrm{~cm}^{-2}\right)$. Polaritons distributed along the 
dispersion curve are monitored by imaging the far field emission of the microcavity on the entrance slit of a monochromator $[19,25,26]$. Emission from the lower polariton ground state at $k_{\|}=0$ is selected with an angular resolution of about $1^{\circ}$ by closing the entrance slit vertically, and sent into the photon autocorrelator. Our autocorrelator is a HBT setup, which consists of a 50:50 nonpolarizing beam splitter and two single photon counters. The photon counters are avalanche photodiodes that have a time resolution as short as $70 \mathrm{ps}$ and a dark count rate of $30 \mathrm{~Hz}$. The time resolution of the photon autocorrelator is $t_{\mathrm{AC}}=120 \mathrm{ps}$ for counting rates lower than $8 \times 10^{5} \mathrm{~s}^{-1}$. In all experiments discussed below, the photon counting rate has been kept below $5 \times 10^{5} \mathrm{~s}^{-1}$.

We have used this autocorrelator to analyze the polariton emission as a function of the excitation intensity. The second-order time correlation function $g^{(2)}(\tau)$ is defined as

$$
g^{(2)}(\tau)=\langle I(t+\tau) I(t)\rangle /[\langle I(t)\rangle\langle I(t+\tau)\rangle],
$$

where $I(t)$ is the polariton emission intensity at time $t$, and $\langle\ldots\rangle$ is time averaged. In a classical Bose gas at thermal equilibrium, such as a photon gas within a black body cavity, the intensity fluctuations are caused by the occupancy fluctuation of a given quantum state which follows a Boltzmann distribution. This distribution leads to $g^{(2)}(0)=$ 2. In our case the situation is more complicated: the polariton gas does not have the time to reach thermal equilibrium because it is leaking very efficiently outside of the microcavity (on a 2 ps time scale). In the low density limit, the statistics of the polariton occupancy is determined by the thermal phonon bath which is responsible for their relaxation. The corresponding value for $g^{(2)}(0)$ thus will reflect that of the phonon bath, modified by the polaritonphonon coupling constant [27]. Then, it will range between $>1$ and 2. Another fact should be taken into account when discussing our measurements: the fluctuations of the polariton field are related to those of the photon field in a nontrivial manner [27,28]. Actually only the coherent state would show the same fluctuations on both the photon and polariton fields, corresponding to $g^{(2)}(0)=1$. On the other hand, if the photon field shows an excess of fluctuations with respect to the fluctuations of a coherent state, we can conclude that the polariton field should show also an excess of fluctuations [but with different value of $g^{(2)}(0)$ ].

We present first correlation measurements in the $\mathrm{cW}$ excitation mode. For low excitations $P \leq P_{\mathrm{thr}}$, a correlation peak of about 3\% height is visible [Fig. 1(a)]. Although low, this correlation peak cannot be mixed up with noise, as it lies 3 times (i.e. with $99 \%$ of confidence level) above the mean noise level measured on the uncorrelated long delay data. This small bunching value must then be corrected to take into account the time resolution of the setup $t_{\mathrm{AC}} \sim 120 \mathrm{ps}$, much longer than the polariton coherence time $t_{C} \sim 2 \mathrm{ps}$ (as independently measured with a Michelson interferometer). In this case, the actual zero delay peak $g^{(2)}(0)$ can be estimated from the measured

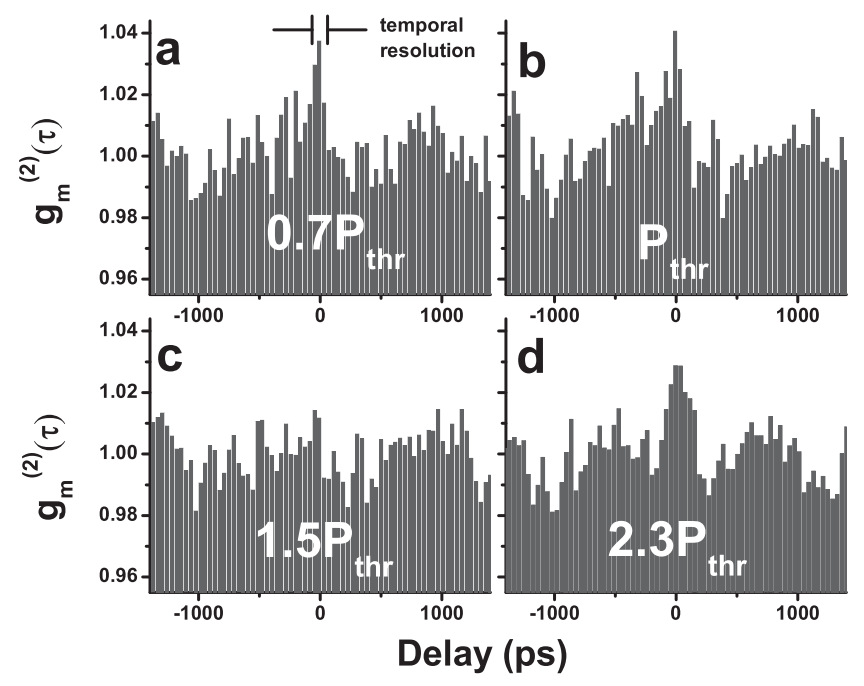

FIG. 1. Second-order time correlations measured for polariton photoluminescence at $k_{\|}=0$, for four different pump intensities. Suppression of the photon bunching is seen as the condensation threshold is passed, demonstrating transition from thermal towards coherent state. Far above threshold (d) the coherence is degraded (photon bunching reappears) due to interactions within the polariton condensate and scatterings with noncondensed excitons and polaritons.

zero delay peak $g_{m}^{(2)}(0)$ by: $g_{m}^{(2)}(0) \approx 1+\left(g^{(2)}(0)-\right.$ 1) $t_{C} / t_{\mathrm{AC}}$. This correction leads to $g^{(2)}(0) \approx 2.8 \pm 1$, which indicates fluctuations significantly higher than those of a coherent state.

At condensation threshold, a significant excess of fluctuations is still present [Fig. 1(b)]. This is not surprising considering the fact that $\mathrm{BEC}$ is a phase transition, and it is common that fluctuations in the photon number of a laser strongly increase at threshold [29]. For excitation $P=$ $1.5 P_{\text {thr }}$, the zero delay fluctuations remain within the noise level of the measurement showing that photon bunching is suppressed [Fig. 1(c)]. This clearly shows that the polariton system is now in a coherent state, and confirms the formation of a Bose condensate as reported in [19]. However, photon bunching reappears at zero time delay with further increase of the excitation [Fig. 1(d)], indicating that coherence in the condensate is degraded at high density. In fact, it is theoretically shown that Coulomb scattering of polaritons within the condensate and with noncondensed polaritons and excitons should rapidly induce phase diffusion in the condensate [22]. Consequently, the condensate emission should be spectrally broadened, which has been indeed observed in the CdTe condensate [19]. In that sense the polariton condensate strongly differs from the photon laser whose coherence increases with the number of photons in the lasing mode.

We turn now to the pulsed excitation measurements. The use of pulsed excitation has some benefits when $g^{(2)}(\tau)$ is to be measured. First, the time resolution of the detectors is no longer an issue, unless it is longer than the excitation pulse period $(\sim 13.1 \mathrm{~ns})$. Second, photons are emitted within a finite time interval after each excitation pulse. 
As a result, for a given counting rate, the number of coincidences is significantly higher than for $\mathrm{cw}$ measurements (by a factor of 20 in our case), making data acquisition times shorter and thus permitting to obtain better signal-to-noise ratio. Finally, it is highly instructive to have a comparison with a previous study of second-order time correlations in a GaAs microcavity which was performed under pulsed excitation [18].

As for the cw study, we use the excitation laser to check the HBT setup in the pulsed mode. The data have been corrected for the "pile-up" effect, which appears when the time delay becomes comparable to the inverse count rate [30]. The error bars in Fig. 2 represent the photon noise $N^{-1 / 2}, N$ being the number of coincidences.

For weak excitation, a small but reproducible photon bunching (about $0.5 \%$ ) is visible at zero time delay [Fig. 2(b)]. This small peak value is due to the fact that photon bunching only occurs during the coherence time $t_{C}$ of polaritons, which is much shorter than their decay dynamics. Although the polariton lifetime is very short the relaxation dynamics could be very slow due to the strong dispersion of the lower polariton branch at wave vectors $k_{\|} \sim 0$, which inhibits acoustic phonon exchange with the lattice. This results in the formation of a reservoir of excitons at large $k_{\|}$(bottleneck effect), which could take several hundreds of picoseconds to relax down to the polariton ground state [18,31-33]. This is directly reflected in the linewidth of the correlation peaks as shown in Fig. 4: At low excitation, the peak is broadened, not by the time resolution of the setup $t_{\mathrm{AC}} \approx 120 \mathrm{ps}$, but by the polariton relaxation dynamics $t_{\text {rel }}(P) \approx 300$ ps. In that case, the measured zero delay peak can be approximated by $g_{m}^{(2)}(0) \approx 1+\left(g^{(2)}(0)-1\right) t_{C} / t_{\text {rel }}(P)$. This correction leads

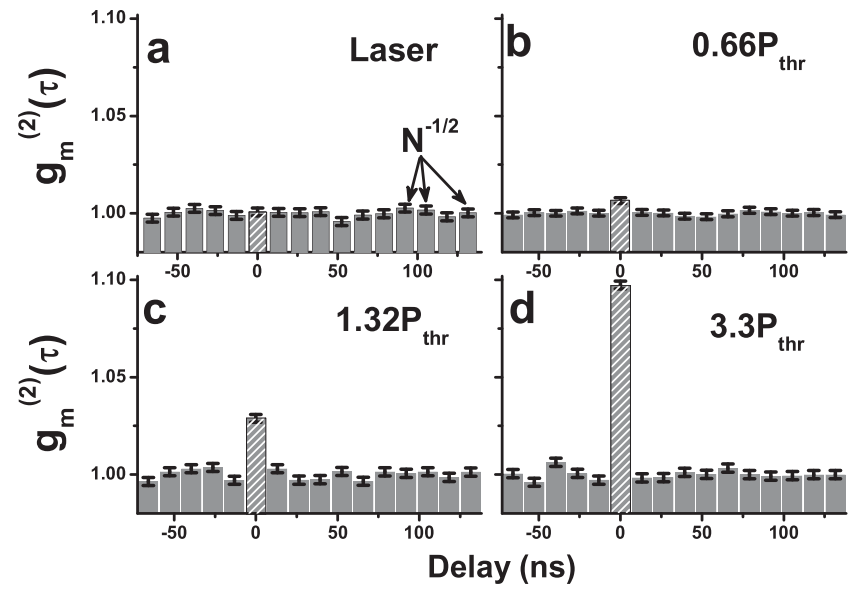

FIG. 2. (a) $g_{m}^{(2)}(\tau)$ measured for the excitation laser. The photon bunching at zero time delay is not observed showing secondorder coherence of the laser field. (b) $-\left(\right.$ d) $g_{m}^{(2)}(\tau)$ measured for the polariton photoluminescence at $k_{\|}=0$. The photon bunching of the order $0.5 \%$ is measured below the condensation threshold. Its value gradually increases with the excitation density (c),(d), and see Fig. 3. The error bars correspond to $N^{-1 / 2}$. to $g^{(2)}(0) \approx 1.8 \pm 0.4$, which is qualitatively in line with the $\mathrm{cw}$ result. When approaching the threshold, the correlation value seems to remain steady, however $t_{\text {rel }}(P)$ is decreasing strongly at the same time. This is visible on the line width decreasing of the zero delay correlation peak (Fig. 4). This accelerated relaxation dynamics has been reported in a detailed time resolved-analysis presented in [31]. This means that the excess of fluctuations is actually dropping while approaching the condensation threshold, consistent with the build up of a coherent state. The decay of the polariton ground state for above threshold excitation always consists of two components: A fast decay corresponding to the condensed phase, and a much slower decay typical of the low excitation regime [18,31-33]. This mixture of coherent and incoherent polaritons explains why, by contrast to the $\mathrm{cw}$ case, photon bunching does not disappear for excitation $P=1.32 P_{\text {thr }}$ [Fig. 2(c)].

Photon bunching increases again with increasing excitation [Fig. 2(d)]. In Fig. 3, we compare $g_{m}^{(2)}(0)$ and the emission linewidth of the polariton ground state as a function of the excitation intensity. A general tendency of decoherence deep in the condensed phase is observed [22], consistent with cw results shown in Fig. 1(d).

The loss of coherence with increasing density in the condensed phase has been observed in both $\mathrm{cw}$ and pulsed experiments in our sample, showing that the general behavior of the polariton condensate does not correspond to the standard photon laser. According to [22], this is a direct

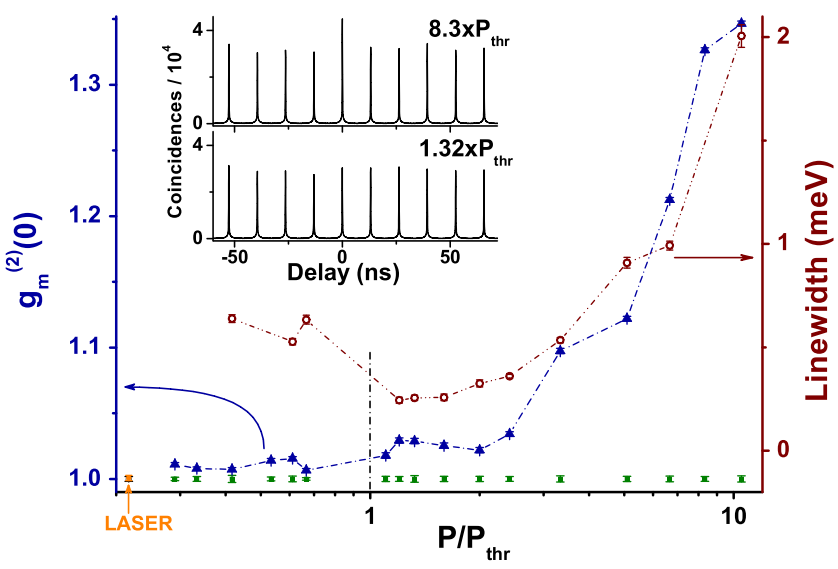

FIG. 3 (color online). $g_{m}^{(2)}(\tau=0)$, for the polariton photoluminescence at $k_{\|}=0$ as a function of the normalized excitation intensity (blue triangles). The $g_{m}^{(2)}(0)$ gradually increases with the pump intensity. In order to interpret this behavior the interplay between the coherence time and the relaxation time should be taken into consideration (see main text). Far above threshold the decoherence is manifested by increase of the linewidth (open brown circles) and by increase of the $g_{m}^{(2)}(0)$. The orange circle is the $g_{m}^{(2)}(0)$ of the excitation laser. Green squares are average values of $g_{m}^{(2)}(\tau \neq 0)$ correlation peaks. Inset: The number of coincidences as a function of $\tau$ measured for the polariton photoluminescence at $k_{\|}=0$ in the pulsed excitation regime; far above threshold (up) a significant photon bunching at $\tau=0$ is measured. 


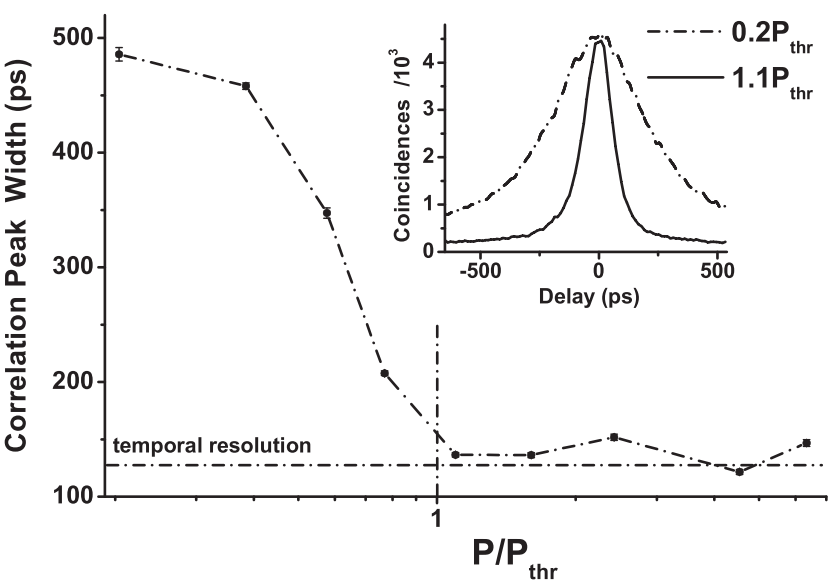

FIG. 4. Acceleration of the polariton relaxation towards the ground state with increasing the pump intensity measured in the intensity correlation experiment. The narrowing of the correlation peaks due to the reduction of the decay time is shown (Inset: below threshold: dashed-dot line and at threshold: solid line). Above condensation threshold the width of the correlation peaks is limited by the $t_{\mathrm{AC}}$ and not by the $t_{\mathrm{rel}}$.

consequence of the excitonic nature of polaritons. On the other hand, a previous study of second-order time correlations in a GaAs microcavity [18] showed that the polariton fluctuations exhibit a slow decrease upon increasing the excitation intensity, from $g^{(2)}(0)=1.77$ at $1.1 P_{\text {thr }}$, to about $g^{(2)}(0)=1.48$ for intensity $\sim 15$ times higher. A possible lead for this discrepancy could stem from the different polariton-polariton interaction strength in GaAs and CdTe. This interaction is proportional to $\lambda_{X} / \epsilon$, where $\lambda_{X}$ is the two-dimensional exciton Bohr radius in QWs, and $\epsilon$ the dielectric constant [34]. Whereas the dielectric constant is about the same in GaAs and CdTe, the exciton Bohr radius in GaAs is typically $150 \AA$, 3 times larger than in CdTe. Thus the polariton-polariton interaction is expected to be 3 times stronger in GaAs, which could prevent the existence of a coherent regime in the condensed phase.

In summary, we have investigated the second-order time correlations of polaritons in a CdTe-based microcavity by a HBT experiment. Results obtained in the $\mathrm{cw}$ excitation mode provide additional support to the BEC recently observed in the same microcavity [19]. Below the condensation threshold, polaritons are in a thermal-like state, yielding photon bunching for zero time delay. As the condensation threshold is passed, photon bunching is suppressed, supporting evidence for the onset of coherence. Increasing further the number of polaritons in the condensed phase results in a loss of coherence, revealed by the appearance of photon bunching and the spectral broadening of the condensate emission. Polaritons are interacting bosons, and phase diffusion is predicted in the polariton condensate at high density due to self interaction [22]. One should also keep in mind the large number of excitons in the reservoir: At threshold, the exciton density in each QW is about $10^{10} \mathrm{~cm}^{-2}$, i.e., 2 orders of magnitude higher than that of polaritons. Therefore interaction with excitons could be a significant source of decoherence and a severe limitation for a practical use of the "polariton laser."

We acknowledge support by the EU Network No. HPRN-CT-2002-00298 "Photon-mediated phenomena in semiconductor nanostructures" and the Swiss National Research Foundation through the "Quantum Photonics NCCR". J.K. would like to acknowledge the UK EPSRC Grant No. EP/D025303/1 for the financial support. We are thankful to Fabrice Donatini for technical assistance.

*Present address: School of Physics and Astronomy, Cardiff University, 5 The Parade, Cardiff, CF24 3AA, United Kingdom.

[1] A. J. Leggett, Rev. Mod. Phys. 73, 307 (2001).

[2] L.P. Pitaevskii and S. Stringari, Bose-Einstein Condensation (Clarendon, Oxford, 2003).

[3] M. H. Anderson et al., Science 269, 198 (1995).

[4] K. B. Davis et al., Phys. Rev. Lett. 75, 3969 (1995).

[5] M. R. Andrews et al., Science 275, 637 (1997).

[6] I. Bloch et al., Nature (London) 403, 166 (2000).

[7] D. Hellweg et al., Phys. Rev. Lett. 91, 010406 (2003).

[8] A. Öttl et al., Phys. Rev. Lett. 95, 090404 (2005).

[9] E. A. Burt et al., Phys. Rev. Lett. 79, 337 (1997).

[10] M. Schellekens et al., Science 310, 648 (2005).

[11] J. L. Lin and J. P. Wolfe, Phys. Rev. Lett. 71, 1222 (1993).

[12] A. Mysyrowicz et al., Phys. Rev. Lett. 77, 896 (1996).

[13] M. Y. Shen et al., Phys. Rev. B 56, 13066 (1997).

[14] L. V. Butov et al., Nature (London) 417, 47 (2002).

[15] L. V. Butov et al., Phys. Rev. Lett. 73, 304 (1994).

[16] J.P. Eisenstein and A.H. MacDonald, Nature (London) 432, 691 (2004).

[17] C. Rüegg et al., Nature (London) 423, 62 (2003).

[18] H. Deng et al., Science 298, 199 (2002).

[19] J. Kasprzak et al., Nature (London) 443, 409 (2006).

[20] C. Weisbuch et al., Phys. Rev. Lett. 69, 3314 (1992).

[21] A. Kavokin and G. Malpuech, Cavity Polaritons (Elsevier, Amsterdam, 2003).

[22] D. Porras and C. Tejedor, Phys. Rev. B 67, 161310(R) (2003).

[23] R. Hanbury Brown and R. Twiss, Nature (London) 177, 27 (1956).

[24] R. André et al., J. Cryst. Growth 184/185, 758 (1998).

[25] M. Richard et al., Phys. Rev. Lett. 94, 187401 (2005).

[26] W. Langbein, Phys. Rev. B 70, 205301 (2004).

[27] J.-P. Karr et al., Phys. Rev. A 69, 031802 (2004).

[28] P. Schwendimann et al., Phys. Rev. B 68, 165324 (2003).

[29] R. Jin et al., Phys. Rev. A 49, 4038 (1994).

[30] In such a case, the HBT setup would register only coincidences from consecutive photons. This slightly differs from the true $g^{(2)}(\tau)$, which should measure correlations between photons even if they are not consecutive.

[31] M. Müller et al., Phys. Rev. B 62, 16886 (2000).

[32] M. Müller et al., Semicond. Sci. Technol. 18, S319 (2003).

[33] J. Bloch et al., Phys. Rev. B 71, 155311 (2005).

[34] C. Ciuti et al., Phys. Rev. B 62, R4825 (2000). 